\title{
2017-18 Congressional Fellowship Program Assignments
}

$\mathrm{F}$ ounded in 1953, the APSA Congressional Fellowship Program is the nation's oldest and most prestigious congressional fellowship. The program remains devoted to its original objective of expanding knowledge and awareness of Congress. For nine months, select political scientists, journalists, doctors, federal executives, and international scholars gain "hands on" understanding of the legislative process by serving on congressional staffs. Through this opportunity, the association enhances public understanding of policy making and improves the quality of scholarship, teaching, and reporting on American national politics. 2017-18 fellows were first announced in the January 2018 issue of $P S$, with full academic and/or professional titles.

POLITICAL SCIENTIST FELLOWS

Emily Baer, PhD

Christopher Baylor, PhD

Rep. Rosa DeLauro (D-CT)

Eric van der Vort, PhD candidate

Rep. Daniel Lipinski (D-IL)

Ryan Williamson, $\mathrm{PhD}$

Carolina Ferrerosa Young, PhD candidate

Rep. Raúl Grijalva (D-AZ)

Senate Committee on Rules and Administration (Minority)

Sen. Mark Warner (D-VA)

JOURNALIST FELLOW

Bridget Balch

Subcommittee on Africa, Global Health, Global Human Rights, and International Organizations, House Foreign Affairs Committee (Majority)

FEDERAL EXECUTIVE FELLOWS

Deborah Hobson

Morgan Large

Summer Lockerbie, PhD

Mathew Stanley

HEALTH \& AGING POLICY FELLOWS

Residential

Carmen Castro, DrPH

Lori Frank, PhD

Lynn Hallarman, MD

Terri D. Lewinson, PhD, MSW

\section{Non-Residential}

M. Batchelor-Murphy, BSN, MSN, PhD

Robert Burke, MD, MS

Anne Fabiny, MD

Adam Golden, MD, MBA

Aaron Leppin, MD, MSc

Ann M. O'Hare, MD

Tiffany Riser, RN, MSN, MPH, ANP-C

Fei Sun, PhD, MSW
Rep. Betty McCollum (D-MN) Rep. Jaime Herrera Beutler (R-WA) Sen. Jack Reed (D-RI) Rep. Bill Posey (R-FL)

\section{ROBERT WOOD JOHNSON FOUNDATION HEALTH POLICY FELLOWS}

Carrie Colla, $\mathrm{PhD}$

Robin Fleming, PhD, MN, RN, NCSN

Caprice Knapp, PhD

Allison Myers, PhD, MPH

Karin Rhodes, MD, MS

Reginald Tucker-Seeley, ScD
Rep. Gene Green (D-TX)

National Institute on Aging Senate Finance Committee (Minority)

Rep. John Lewis (D-GA)

\section{SASAKAWA PEACE FOUNDATION USA FELLOW}

Koki Fukasaku, Associate, Matsushita Institute of Government and Management

Senate Special Committee on Aging (Majority) Center for Medicare and Medicaid Services - Federal Coordinated Health Care Office Project between San Francisco Veterans Affairs Health Care System, the California Medicaid Office and the Health Plan of San Mateo County Center for Evidence and Practice Improvement, Agency for Healthcare Research and Quality Minnesota Board on Aging Center for Medicare \& Medicaid Innovation, Centers for Medicare \& Medicaid Services Congressional Research Service World Health Organization 